\title{
Neural Network-Based Nonlinear Fixed-Time Adaptive Practical Tracking Control for Quadrotor Unmanned Aerial Vehicles
}

\author{
Jianhua Zhang $\mathbb{D}^{1}{ }^{1}$ Yang Li, ${ }^{1}$ and Wenbo Fei ${ }^{2}$ \\ ${ }^{1}$ School of Information and Control Engineering, Qingdao University of Technology, Qingdao, Shandong 266525, China \\ ${ }^{2}$ Hebei University of Science and Technology, Shijiazhuang, Hebei 050018, China \\ Correspondence should be addressed to Jianhua Zhang; jianhuazhang@aliyun.com
}

Received 20 August 2020; Revised 5 September 2020; Accepted 11 September 2020; Published 26 September 2020

Academic Editor: Shubo Wang

Copyright ( 12020 Jianhua Zhang et al. This is an open access article distributed under the Creative Commons Attribution License, which permits unrestricted use, distribution, and reproduction in any medium, provided the original work is properly cited.

This brief addresses the position and attitude tracking fixed-time practical control for quadrotor unmanned aerial vehicles (UAVs) subject to nonlinear dynamics. First, by combining the radial basis function neural networks (NNs) with virtual parameter estimating algorithms, a NN adaptive control scheme is developed for UAVs. Then, a fixed-time adaptive law is proposed for neural networks to achieve fixed-time stability, and convergence time is dependent only on control gain parameters. Based on Lyapunov analyses and fixed-time stability theory, it is proved that the fixed-time adaptive neural network control is finite-time stable and convergence time is dependent with control parameters without initial conditions. The effectiveness of the NN fixedtime control is given through a simulation of the UAV system.

\section{Introduction}

During the last decades, more attention has been paid to UAVs by the engineering communities because of their potential for commercial, military, and academic platforms [1-3]. To support military and civilian applications, the ability of UAVs to hover stably, maneuver sharply, and operate safely is considerably important, especially in takeoff and landing stages [4-6]. However, tracking control design for UAVs in complex environments is challenging because of the nonlinear dynamics of UAVs. In order to meet various application requirements, especially military requirements, UAVs are required to have better robustness, stability, and fast maneuverability [7]. Drones have a wealth of applications in various fields, such as monitoring, spraying pesticides, and delivering couriers.

Since the advent of the first rotorcraft in the twentieth century, in order to solve the impact of the actual situation and better complete the motion control of the four-rotor UAV, scholars have published numerous documents to solve a series of problems. With the advancement of science and the development of NNs [8-10], NNs have been widely used and verified in the quadrotor intelligent control. As we all know, the neural network has been questioned by some scholars in the long history since it was proposed, but it is finally proved after research by scholars in many fields that the neural network is an advantageous control algorithm [11-13]. Although neural networks have long been used in the quadrotor intelligent control and have achieved good results in many research studies, the research is not comprehensive. In recent years, the study of finite time adaptive neural networks has been carried out by scholars, and a fixed-time control is proposed to solve the dependence of convergence on the initial state. In this paper, the nonlinear fixed-time adaptive neural network control of the quadcopter UAV is studied.

Although the quadrotor has many advantages, due to the nonlinearity, coupling, underdrive, and susceptibility to interference of the dynamics of the UAV, it is necessary to develop tracking control in uncertain environments. In the past few years, the authors studied the design of the dynamic mathematic model of the UAV in [14], as well as dynamic control of the UAV. In [15-17], the authors proposed a PID/ PD control method to maintain flight stability, but due to the shortcomings of the algorithm, the authors designed a neural network adaptive PID algorithm in [18]. In order to 
solve the underdrive of the quadrotor, the authors proposed four additional control inputs in [19] to represent a fully driven aircraft. In [20], the authors used sliding film control. The quadrotor is susceptible to a variety of unknown disturbances during flight. In [21-23], the authors designed a variety of controllers to stabilize the disturbances, but they were not perfect. Backstepping control design has been widely used $[24,25]$ for a class of nonlinear system control. The four-rotor aircraft has obtained relevant research results during take-off, hover, and landing [26-28]. The interference caused by machine failure cannot be avoided during flight. In [29], the authors proposed a method to solve the loss of control effectiveness of the aircraft through adaptive control and quantum logic. It is well known that neural network algorithms have advanced control performance. In [30, 31], the authors proposed neural network algorithms to control flight attitude and position. Some researchers also used NNs to model a quadrotor aircraft in [32], which can more accurately describe the quadrotor model. In order to ensure the tracking error, the inversion controller was used in [33] to study the adaptive flight control neural network and dynamic inversion control. Quadrotor flight control researchers have proposed a wide variety of control algorithms. In [34], the authors proposed dynamic feedback linearization of a four-axis aircraft for chart learning. In order to enhance the stability of the UAV, the author applied the feedforward control method in [35]. Finite-time control $[36,37]$ plays an important role in solving the convergence time problem.

In this paper, practical fixed-time adaptive $\mathrm{NN}$ control for the UAV system is proposed. The closed-loop UAV position and attitude tracking error system is practically fixed-time stable, whereas the convergence time is independent of initial states and tracking error is convergence to a small neighborhood of the origin point. The major contributions of this brief can be stated as follows:
(1) Based on NNs adaptive control and practical fixedtime stability theory, the controller parameters for the UAVs position and attitude tracking are designed.

(2) The fixed-time adaptive law is designed for NNs, and error weight is practically stable in finite time. Therefore, the NNs can approximate the nonlinear system in finite time independent of ideal weight and initial value of estimated weight.

(3) To avoid the finite-time control singular problem in the high-order system, a switch virtual control law is proposed to guarantee the feasibility of the fixedtime control.

\section{Preliminary Problem Formulation}

A dynamic model $[30,38]$ is established according to the motion laws of the four-rotor power system; the body coordinate system $B: O_{b} X_{b} Y_{b} Z_{b}$ and the ground coordinate systemE: $O_{e} X_{e} Y_{e} Z_{e}$ are selected, as shown in Figure 1. In the Newton-Euler formula, $[x, y, z]^{T}$ indicates the Euclidean position and $[\phi, \theta, \psi]$ indicates the Euler angles of UAVs. When the four-rotor drone changes its motion, the body coordinate system will change continuously with the drone and the ground coordinate system will remain constant. Due to the need to analyze the problem in the ground coordinate system, the spatial rotation of the body coordinate system relative to the geographic coordinate system is usually expressed in the order of yaw angle, pitch angle, and roll angle. The matrix is used to describe the yaw angle $\psi$, pitch angle $\theta$, and roll angle $\phi$. Based on the relationship between the angles in the coordinate system $B$ and the coordinate system $E$, the coordinate transformation matrix can be obtained.

The transformation from body coordinates to ground coordinates is performed by the rotation matrix:

$$
R=\left[\begin{array}{ccc}
\cos \theta \cos \psi & \cos \psi \sin \theta \sin \phi-\cos \phi \sin \psi & \cos \phi \cos \psi \sin \theta+\sin \phi \sin \psi \\
\cos \theta i n \psi & \sin \theta \sin \phi \sin \psi+\cos \phi \cos \psi & \cos \phi \sin \theta i n \psi-\cos \psi \sin \phi \\
-\sin \theta & \cos \theta \sin \phi & \cos \theta \cos \phi
\end{array}\right]
$$

When modeling the quadrotor as a rigid body with uniform mass distribution, symmetry, and constant, according to Newton's second law:

$$
F_{\mathrm{all}}=m\left[\begin{array}{c}
\ddot{x} \\
\ddot{y} \\
\ddot{z}
\end{array}\right]
$$

where $m$ is the weight of the quadrotor drone and $\ddot{x}, \ddot{y}$, and $\ddot{z}$ are the acceleration in three directions, and the force $F_{\text {all }}$ in three directions is obtained.

According to the force analysis of the drone in motion, it can be known that the external force received has its own gravity $G$, the lift force $F_{\text {at }}$ generated by the rotor, and the rising resistance $f_{t}$ :

$$
\begin{aligned}
G & =m g, \\
F_{\text {at }} & =k \omega_{j}^{2}(j=1,2,3,4), \\
{\left[\begin{array}{l}
f_{x} \\
f_{y} \\
f_{z}
\end{array}\right] } & =\left[\begin{array}{l}
\xi_{x} \dot{x} \\
\xi_{y} \dot{y} \\
\xi_{z} \dot{z}
\end{array}\right] .
\end{aligned}
$$

According to equation (3), the centroid motion equation of the quadrotor UAV with the positive $Z$ axis in the ground coordinate system can be obtained: 


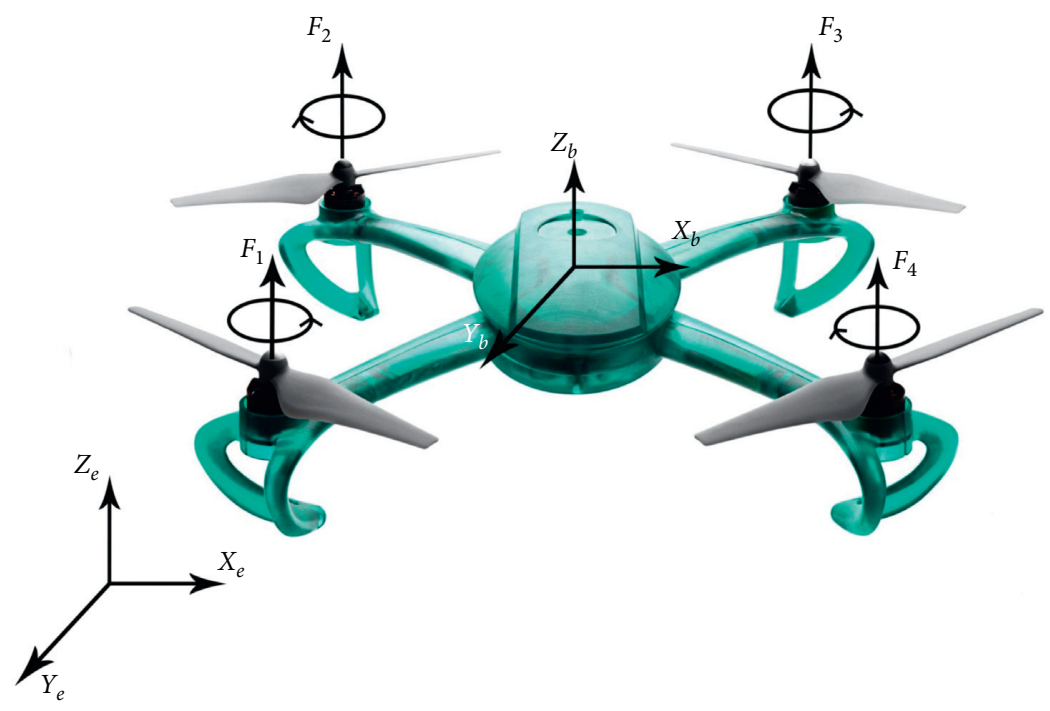

FIGURE 1: Schematic of the quadrotor system.

$$
m[\ddot{x} \ddot{y} \ddot{z}]=R F_{\text {at }}\left[\begin{array}{l}
0 \\
0 \\
1
\end{array}\right]-m g\left[\begin{array}{l}
0 \\
0 \\
1
\end{array}\right]-\left[\begin{array}{c}
\xi_{x} \dot{x} \\
\xi_{y} \dot{y} \\
\xi_{z} \dot{z}
\end{array}\right] .
$$

To sum up, we can get the centroid motion equations of the quadrotor UAV as

$$
\left\{\begin{array}{l}
x=\frac{F_{\text {at }}}{m}(\sin \psi \sin \phi+\cos \psi \sin \theta \cos \phi)-\frac{\xi_{x}}{m} \dot{x}, \\
y=\frac{F_{\text {at }}}{m}(-\cos \psi \sin \phi+\sin \phi \sin \theta \cos \psi)-\frac{\xi_{y}}{m} \dot{y}, \\
z=\frac{F_{\text {at }}}{m}(\cos \theta \cos \phi)-g-\frac{\xi_{z}}{m} \dot{z},
\end{array}\right.
$$

where $k$ is the motor pulling force coefficient, $\omega_{j}(j=1,2,3,4)$ is the motor speed, and $\xi_{i}(i=x, y, z)$ is the air resistance coefficient.

Four-rotor drones are divided into cross-shaped structure and X-shaped structure. Due to the difference in structure, the rotation dynamic equation of the drone will also be different. The cross-shaped structure is selected to build a model based on the Newton-Euler equation:

$$
\bar{M}=I \tau+\bar{w} \times I \bar{w}=\left[\begin{array}{ccc}
I_{x} & 0 & 0 \\
0 & I_{y} & 0 \\
0 & 0 & I_{z}
\end{array}\right]\left[\begin{array}{c}
\dot{p} \\
\dot{q} \\
\dot{r}
\end{array}\right]+\left[\begin{array}{c}
\left(I_{z}-I_{y}\right) q r \\
\left(I_{x}-I_{z}\right) r p \\
\left(I_{y}-I_{x}\right) p q
\end{array}\right],
$$

and then

$$
\bar{M}=\left[\begin{array}{c}
M_{x} \\
M_{y} \\
M_{z}
\end{array}\right]=\left[\begin{array}{l}
\dot{p} I_{x}+\left(I_{z}-I_{y}\right) q r \\
\dot{q} I_{y}+\left(I_{x}-I_{z}\right) r p \\
\dot{r} I_{z}+\left(I_{y}-I_{x}\right) p q
\end{array}\right] .
$$

Among them, $\bar{M}$ is the combined external moment on the body, $I=\operatorname{diag}\left(I_{x}, I_{y}, I_{z}\right)$ is the moment of inertia of the three coordinate axes in the body coordinate system, and $\bar{w}=\operatorname{diag}(p, q, r)$ is the rotational angular velocity in the body coordinate system. The force analysis of the moving four-rotor UAV shows that there are torque generated by the rotor, air reaction force, and gyro effect.

The moment produced by the rotor is as follows:

$$
\left\{\begin{array}{l}
u_{1}=F_{a t}=k\left(\omega_{1}^{2}+\omega_{2}^{2}+\omega_{3}^{2}+\omega_{4}^{2}\right), \\
u_{2}=\tau_{a \phi}=k l\left(\omega_{3}^{2}-\omega_{1}^{2}\right), \\
u_{3}=\tau_{a \theta}=k l\left(\omega_{4}^{2}-\omega_{2}^{2}\right), \\
u_{4}=\tau_{a \psi}=b\left(\omega_{2}^{2}-\omega_{1}^{2}+\omega_{4}^{2}-\omega_{3}^{2}\right),
\end{array}\right.
$$

where $l$ is the distance between the center of mass and the rotor axis, $b$ is the drag coefficient, $k$ is the reverse torque coefficient, and $\omega$ is the motor speed.

The air reaction force is as follows:

$$
\tau_{f}=\xi_{k} w,
$$

where $\xi_{k}=\operatorname{diag}\left(\begin{array}{lll}\xi_{\phi} & \xi_{\theta} & \xi_{\psi}\end{array}\right)$ is the air drag moment coefficient in the airframe coordinate system and $w$ is the rotor angular velocity.

The torque of the quadcopter is as follows:

$$
\mathrm{E}_{f}=I_{r} w \omega,
$$

where $I_{r}$ is the rotor inertia, $w$ is the rotor angular velocity, and $\Phi=\omega_{4}+\omega_{2}-\omega_{3}-\omega_{1}$ is the overall remaining rotor angle.

From Newtonian mechanical analysis and equations (8)-(10), we get 


$$
\left[\begin{array}{c}
M_{x} \\
M_{y} \\
M_{z}
\end{array}\right]=\left[\begin{array}{c}
\dot{p} I_{x}+\left(I_{z}-I_{y}\right) q r \\
\dot{q} I_{y}+\left(I_{x}-I_{z}\right) r p \\
\dot{r} I_{z}+\left(I_{y}-I_{x}\right) p q
\end{array}\right]=\left[\begin{array}{c}
\tau_{a \phi}-\xi_{\phi} \dot{\phi}-I_{r} \dot{\theta} \omega \\
\tau_{a \theta}-\xi_{\theta} \dot{\theta}-I_{r} \dot{\phi} \omega \\
\tau_{a \psi}-\xi_{\psi} \dot{\psi}
\end{array}\right]
$$

In summary, when the rotation angle is small, the dynamic model of the quadrotor can be obtained as

$$
\left\{\begin{array}{l}
x=\frac{F_{\text {at }}}{m}(\sin \psi \sin \phi+\cos \psi \sin \theta \cos \phi)-\frac{\xi_{x}}{m} \dot{x}, \\
y=\frac{F_{\text {at }}}{m}(-\cos \psi \sin \phi+\sin \psi \sin \theta \cos \phi)-\frac{\xi_{y}}{m} \dot{y}, \\
z=\frac{F_{\text {at }}}{m}(\cos \theta \cos \phi)-g-\frac{\xi_{z}}{m} \dot{z}, \\
\ddot{\phi}=\dot{\theta} \dot{\psi} \frac{\left(I_{y}-I_{z}\right)}{I_{x}}+\frac{\tau_{a \phi}}{I_{x}}-\frac{I_{r}}{I_{x}} \dot{\theta} \omega-\frac{\xi_{\phi}}{I_{x}} \dot{\phi}, \\
\ddot{\theta}=\dot{\phi} \dot{\psi} \frac{\left(I_{z}-I_{x}\right)}{I_{y}}+\frac{\tau_{a \theta}}{I_{y}}-\frac{I_{r}}{I_{y}} \dot{\phi} \omega-\frac{\xi_{\theta}}{I_{x}} \dot{\theta}, \\
\ddot{\psi}=\dot{\theta} \dot{\phi} \frac{\left(I_{x}-I_{y}\right)}{I_{z}}+\frac{\tau_{a \psi}}{I_{z}}-\frac{\xi_{\psi}}{I_{x}} \dot{\psi} .
\end{array}\right.
$$

In order to facilitate the design of the controller, the dynamic model of the quadrotor flight was rewritten into a compact dynamic equation. $d_{a}(\cdot)=\operatorname{diag}$ $\left[\begin{array}{lll}d_{x}(\cdot) & d_{y}(\cdot) & \left.d_{z}(\cdot)\right]\end{array}\right.$ and $d_{p}(\cdot)=\operatorname{diag}\left[d_{\phi}(\cdot) d_{\theta}(\cdot) d_{\psi}(\cdot)\right]$ take into account the external disturbance of the position and attitude of the quadrotor in motion.

(1) Translational dynamics:

$$
\ddot{x}=A u_{s}(t)+f_{1}(\cdot)+d_{a}(x, t) .
$$

(2) Rotational dynamics:

$$
\ddot{p}=B u_{r}(t)+f_{2}(\cdot)+d_{b}(p, t),
$$

where

$$
A=\left[\begin{array}{ccc}
\frac{1}{m} & 0 & 0 \\
0 & \frac{1}{m} & 0 \\
0 & 0 & \frac{1}{m}
\end{array}\right],
$$

$$
B=\left[\begin{array}{ccc}
\frac{1}{I_{x}} & 0 & 0 \\
0 & \frac{1}{I_{y}} & 0 \\
0 & 0 & \frac{1}{I_{z}}
\end{array}\right],
$$$$
f_{2}(\cdot)=\left(\begin{array}{c}
\frac{I_{y}-I_{z}}{I_{x}} \dot{\theta} \dot{\psi}-\frac{I_{r}}{I_{x}} \dot{\theta} \omega-\frac{\xi_{\phi}}{I_{x}} \\
\frac{I_{z}-I_{x}}{I_{y}} \dot{\phi} \dot{\psi}-\frac{I_{r}}{I_{y}} \dot{\phi} \omega-\frac{\xi_{\theta}}{I_{y}} \\
-\frac{\xi_{\psi}}{I_{z}}
\end{array}\right),
$$

$$
f_{1}(\cdot)=\left(\begin{array}{c}
-\frac{\xi_{x}}{m} \dot{x} \\
-\frac{\xi_{y}}{m} \dot{y} \\
-\frac{\xi_{z}}{m} \dot{z}-g
\end{array}\right) \text {, }
$$

$u_{s}(t)==\left[\begin{array}{c}(\sin \psi \sin \phi+\cos \psi \sin \theta \cos \phi) u_{1} \\ (-\cos \psi \sin \phi+\sin \psi \sin \theta \cos \phi) u_{1} \\ (\cos \theta \cos \phi) u_{1}\end{array}\right]$,

$u_{r}(t)=\left[\begin{array}{l}u_{2} \\ u_{3} \\ u_{4}\end{array}\right]$ 
Lemma 1 (see $[30,39,40]$ ). Suppose that $V(\cdot): R^{n} \longrightarrow$ $R_{+} \cup\{0\}$ is a continuous radically unbounded function and the following two conditions hold

$$
\begin{aligned}
& V(x)=0 \Longleftrightarrow x=0, \\
& \dot{V}(x) \leq-a V(x)^{p}-b V(x)^{q}+c,
\end{aligned}
$$

where $a, b, p$, and $q$ are the positive real numbers with $p \in(0,1)$ and $q \in(1, \infty)$. Then, the origin $x=0$ of the system $\dot{x}=f(t, x)$ and $x(0)=x_{0}$ is practically fixed-time stable. Furthermore, the following inequality holds:

$$
V(x, t) \leq \xi, t \geq T_{\max },
$$

where $\xi$ is the equation roots, and

$$
\begin{aligned}
2^{p-1} a \xi^{p}+b \xi^{q} & =c, \\
T_{\max } & =\frac{1}{2^{p-1} a(1-p)}+\frac{1}{b(q-1)} .
\end{aligned}
$$

Lemma 2 (see [39]). For $x_{i} \in R$ and $x_{i} \geq 0, i=1,2, \cdots, n$, $0<p<1$, and $q>1$, and then

$$
\begin{aligned}
& \left(\sum_{i=1}^{n} x_{i}\right)^{p} \leq \sum_{i=1}^{n} x_{i}^{p} \leq n^{1-p}\left(\sum_{i=1}^{n} x_{i}\right)^{p}, \\
& n^{1-q}\left(\sum_{i=1}^{n} x_{i}\right)^{q} \leq \sum_{i=1}^{n} x_{i}^{q} \leq\left(\sum_{i=1}^{n} x_{i}\right)^{q} .
\end{aligned}
$$

Proof. Inequalities (19) and (20) hold trivially for

$$
x_{1}=x_{2}=\cdots x_{n}=0 .
$$

For inequality (19), using the fact $x^{p} \geq x$ for $\forall x \in(0,1]$ and $0<p \leq 1$, we have

$$
\frac{\sum_{i=1}^{n} x_{i}^{p}}{\left(\sum_{i=1}^{n} x_{i}\right)^{p}}=\sum_{i=1}^{n}\left(\frac{x_{i}}{\sum_{i=1}^{n} x_{i}}\right)^{p} \geq \sum_{i=1}^{n}\left(\frac{x_{i}}{\sum_{i=1}^{n} x_{i}}\right)=1,
$$

which proves the inequality

$$
\left(\sum_{i=1}^{n} x_{i}\right)^{p} \leq \sum_{i=1}^{n} x_{i}^{p}
$$

Based on the Holder inequality,

$$
\sum_{i=1}^{n} x_{i}^{p} \eta_{i} \leq\left(\sum_{i=1}^{n}\left(x_{i}^{p}\right)^{(1 / p)}\right)^{p}\left(\sum_{i=1}^{n}\left(\eta_{i}\right)^{(1 / 1-p)}\right)^{1-p},
$$

for $\forall x_{i}, \eta_{i}>0$, and let $\eta_{i}=1, i=1,2, \cdots, n$,

$$
\sum_{i=1}^{n} x_{i}^{p} \leq n^{1-p}\left(\sum_{i=1}^{n} x_{i}\right)^{p}
$$

which proved inequality (19).

Based on the Holder inequality,

$$
\sum_{i=1}^{n} x_{i} \eta_{i} \leq\left(\sum_{i=1}^{n} x_{i}^{q}\right)^{(1 / q)}\left(\sum_{i=1}^{n} \eta_{i}^{(q / q-1)}\right)^{(q-1 / q)},
$$

for $\forall x_{i}, \eta_{i}>0$, and let $\eta_{i}=1, i=1,2, \cdots, n$, which proved the inequality holds

$$
n^{1-q}\left(\sum_{i=1}^{n} x_{i}\right)^{q} \leq \sum_{i=1}^{n} x_{i}^{q}
$$

For inequality (20) using the fact $x^{q} \leq x$ for $\forall x \in(0,1]$ and $q>1$, we have

$$
\frac{\sum_{i=1}^{n} x_{i}^{q}}{\left(\sum_{i=1}^{n} x_{i}\right)^{q}}=\sum_{i=1}^{n}\left(\frac{x_{i}}{\sum_{i=1}^{n} x_{i}}\right)^{q} \leq \sum_{i=1}^{n}\left(\frac{x_{i}}{\sum_{i=1}^{n} x_{i}}\right)=1,
$$

which proves the inequality

$$
\sum_{i=1}^{n} x_{i}^{q} \leq\left(\sum_{i=1}^{n} x_{i}\right)^{q}
$$

which proved inequality (20).

Lemma 3 (see [39]) (Young's inequality). For any constant $a, b \in \mathfrak{R}$, the following inequality holds:

$$
a b \leq \frac{1}{p} a^{p}+\frac{1}{q} b^{q}
$$

where $p>1, q>1$, and $(1 / p)+(1 / q)=1$.

Lemma 4 (see [1]). For positive constant $\eta=(1 / 3)$, the vector quantities $W^{*}, \widehat{W}, \widetilde{W} \in \mathfrak{R}^{m}$ and satisfy $\widetilde{W}=\widehat{W}-W^{*}$ and the following inequality holds:

$$
-\tilde{W}^{T} \widehat{W}^{\eta} \leq-\frac{1}{2} \tilde{W}^{(\eta+1 / 2) T} \tilde{W}^{(\eta+1 / 2)}+W^{*(\eta+1 / 2) T} W^{*(\eta+1 / 2)} .
$$

Proof. For any constant $\widetilde{\omega}_{i}, \widehat{\omega}_{i}, \omega_{i}^{*} \in \mathfrak{R}, \widetilde{W}=\left[\widetilde{\omega}_{1} \widetilde{\omega}_{2} \cdot \widetilde{\omega}_{m}\right]^{T}$, $\widehat{W}=\left[\begin{array}{llll}\widehat{\omega}_{1} & \widehat{\omega}_{2} & \ldots & \widehat{\omega}_{m}\end{array}\right]^{T}, \quad W^{*}=\left[\begin{array}{llll}\omega_{1}^{*} & \omega_{2}^{*} & \ldots & \omega_{m}^{*}\end{array}\right]^{T}$, and $\widehat{W}^{\eta}=\left[\begin{array}{llll}\widehat{\omega}_{1}^{\eta} & \widehat{\omega}_{2}^{\eta} & \ldots & \widehat{\omega}_{m}^{\eta}\end{array}\right]^{T}$, based on Lemma 3 (Young's inequation), the following inequations hold:

$$
\begin{gathered}
-\widetilde{\omega}_{i}^{3} \omega_{i}^{*} \leq \frac{3}{4} \widetilde{\omega}_{i}^{4}+\frac{1}{4} \omega_{i}^{* 4} \\
\frac{3}{2} \widetilde{\omega}_{i}^{(4 / 3)} \omega_{i}^{*(8 / 3)} \leq \frac{3}{4} \widetilde{\omega}_{i}^{(8 / 3)} \omega_{i}^{*(4 / 3)}+\frac{3}{4} \omega_{i}^{* 4} .
\end{gathered}
$$

Based on inequations (32) and (33), it gives

$$
-\widetilde{\omega}_{i}^{4}-\widetilde{\omega}_{i}^{3} \omega_{i}^{*} \leq-\frac{1}{8} \widetilde{\omega}_{i}^{4}+\omega_{i}^{* 4}+\frac{3}{4} \widetilde{\omega}_{i}^{(8 / 3)} \omega_{i}^{*(4 / 3)}-\frac{3}{2} \widetilde{\omega}_{i}^{(4 / 3)} \omega_{i}^{*(8 / 3)} \text {. }
$$

Therefore,

$$
-\widetilde{\omega}_{i}^{3}\left(\widetilde{\omega}_{i}+\omega_{i}^{*}\right) \leq\left(-\frac{1}{2} \widetilde{\omega}_{i}^{(4 / 3)}+\omega_{i}^{*(4 / 3)}\right)^{3},
$$

and then 


$$
-\widetilde{\omega}_{i} \widehat{\omega}_{i}^{(1 / 3)} \leq-\frac{1}{2} \widetilde{\omega}_{i}^{(4 / 3)}+\omega_{i}^{*(4 / 3)}
$$

Therefore,

$$
-\sum_{i=1}^{m} \widetilde{\omega}_{i} \widehat{\omega}_{i}^{(1 / 3)} \leq-\frac{1}{2} \sum_{i=1}^{m} \widetilde{\omega}_{i}^{(4 / 3)}+\sum_{i=1}^{m} \omega_{i}^{*(4 / 3)},
$$

and then the following inequation holds:

$$
-\widetilde{W}^{T} \widehat{W}^{(1 / 3)} \leq-\frac{1}{2} \widetilde{W}^{(2 / 3) T} \widetilde{W}^{(2 / 3)}+W^{*(2 / 3) T} W^{*(2 / 3)} .
$$

This is the proof.

Notation 1. To avoid control singularity problem in virtual control, if $p<1$, then design $\xi^{p}$ is chosen as

$$
\xi^{p}= \begin{cases}\xi^{p}, & |\xi| \geq \varepsilon, \\ \varepsilon^{p-1} \xi, & |\xi|<\varepsilon .\end{cases}
$$

Therefore, $\xi^{p}$ is continuous and differentiable.

Let rational number $\eta$, matrix $W \in \mathfrak{R}^{m \times n}$, and matrix $W^{\eta}$ denote element-by-element powers, where

$$
\begin{aligned}
W & =\left[\begin{array}{cccc}
w_{11} & w_{12} & \cdots & w_{1 n} \\
w_{21} & w_{22} & \cdots & w_{2 n} \\
\vdots & \vdots & \ddots & \vdots \\
w_{m 1} & w_{m 2} & \cdots & w_{m n}
\end{array}\right], \\
W^{\eta} & =\left[\begin{array}{cccc}
w_{11}^{\eta} & w_{12}^{\eta} & \cdots & w_{1 n}^{\eta} \\
w_{21}^{\eta} & w_{22}^{\eta} & \cdots & w_{2 n}^{\eta} \\
\vdots & \vdots & \ddots & \vdots \\
w_{m 1}^{\eta} & w_{m 2}^{\eta} & \cdots & w_{m n}^{\eta}
\end{array}\right] .
\end{aligned}
$$

$\tilde{W}^{\eta T}$ denotes the transposition of matrix $\widetilde{W}^{\eta}$, where

$$
\tilde{W}^{\eta T}=\left(\tilde{W}^{\eta}\right)^{T}
$$

\section{Neural Network Control}

Based on the dynamic model of the quadrotor (12), the translational and rotational tracking errors are defined as

$$
\begin{aligned}
& e_{x}=x-x_{d}, \\
& e_{p}=p-p_{d} .
\end{aligned}
$$

To facilitate the control design, the derivation of translational and rotational tracking errors is as follows:

$$
\begin{aligned}
& s_{1}=\dot{x}-\dot{x}_{d}, \\
& s_{2}=\dot{p}-\dot{p}_{d} .
\end{aligned}
$$

Therefore, the translational and rotational tracking error control system is as follows:

$$
\left\{\begin{array}{l}
\dot{e}_{x}=s_{1}, \\
\dot{s}_{1}=A u_{s}(t)+f_{1}(\cdot)+d_{a}(t)-\ddot{x}_{d}
\end{array},\right.
$$

$$
\left\{\begin{array}{l}
\dot{e}_{p}=s_{2}, \\
\dot{s}_{2}=B u_{r}(t)+f_{2}(\cdot)+d_{p}(t)-\ddot{p}_{d}
\end{array} .\right.
$$

Theorem 1. Considering the quadrotor UAV system (12), the translational dynamic system (13), and the translational error control system (44), the fixed-time control parameters $p=(1 / 3)$ and $q=3$ and the virtual control are designed as

$$
\alpha_{\xi}=-a_{1} \xi_{1}^{p}-b_{1} \xi_{1}^{q}, \quad a_{1}>0, b_{1}>0
$$

The fixed-time neural network adaptive control can be designed as

$$
u_{s}=A^{-1}\left(-\widehat{W}_{1}^{T} \Psi_{x}-\ddot{x}_{d}+\dot{\alpha}_{\xi}-\xi_{1}-a_{2} \xi_{2}^{p}-b_{2} \xi_{2}^{q}\right), \quad a_{2}>0, b_{2}>0 .
$$

With the neural fixed-time adaptive law,

$$
\dot{\hat{W}}_{1}=\Gamma_{1}\left(\Psi_{x} \xi_{2}-\sigma_{a x} \widehat{W}_{1}^{p}-\sigma_{b x} \widehat{W}_{1}^{q}\right), \quad \Gamma_{1}>0, \sigma_{a x}>0, \sigma_{a x}>0 .
$$

The translational dynamic system is fixed-time stable and the fixed time is $T_{x \max }=\left(2^{(3-p / 2)} /(1-p) a_{x}\right)+$ $\left(2 /(q-1) b_{x}\right)$.

For the rotational dynamic system (14), the desired roll and pitch references are obtained from

$$
\begin{aligned}
& \phi_{d}=\arcsin \left(\frac{Q_{x} \sin \left(\psi_{d}\right)-Q_{y} \cos \left(\psi_{d}\right)}{\left\|u_{s}\right\|}\right), \\
& \theta_{d}=\arctan \left(\frac{Q_{x} \cos \left(\psi_{d}\right)+Q_{y} \sin \left(\psi_{d}\right)}{Q_{z}}\right) .
\end{aligned}
$$

Based on the translational errors control system (45), the virtual control is designed as

$$
\alpha_{\zeta}=-a_{3} \zeta^{p}-b_{3} \zeta^{q}
$$

and the fixed-time neural network adaptive control can be designed as

$$
u_{r}=B^{-1}\left(-\widehat{W}_{2}^{T} \Psi-\ddot{p}_{d}+\dot{\alpha}_{\zeta}-\zeta_{1}-a_{4} \zeta_{2}^{p}-b_{4} \zeta_{2}^{q}\right) .
$$

With the neural fixed-time adaptive law,

$$
\dot{\hat{W}}_{2}=\Gamma_{2}\left(\Psi_{p} \zeta_{2}-\sigma_{a p} \widehat{W}_{2}^{p}-\sigma_{b p} \widehat{W}_{2}^{q}\right)
$$

The rotational dynamic system is fixed-time stable and the fixed time is $T_{p \max }=\left(2^{(3-p / 2)} /(1-p) a_{p}\right)+$ $\left(2 /(q-1) b_{p}\right)$.

Then, the error translational and rotational dynamic closed-loop systems are practically fixed-time stable; the output tracking error and error of estimate weights can converge to the origin in finite time, and all the signals in the closed-loop system are bounded. 
Proof. The proof of the sufficiency of Theorem 1 is divided into two parts. The first part gives translational dynamic system controller design and stability analysis, and the second one proposes rotational dynamic system controller design and stability analysis.

Step 1. According to the translational tracking error control system, let

$$
\xi_{1}=e_{x},
$$

and then based on (44), we have

$$
\dot{\xi}_{1}=-a_{1} \xi_{1}^{p}-b_{1} \xi_{1}^{q}+\xi_{2}
$$

where

$$
\xi_{2}=s_{1}-\alpha_{\xi},
$$

and then we have

$$
\begin{aligned}
\dot{\xi}_{2} & =A u_{s}+f_{1}+d_{a}-x_{d}-\dot{\alpha}_{\xi} \\
& =A u_{s}+W_{1}^{* T} \Psi_{x}+\varepsilon_{x}-x_{d}-\dot{\alpha}_{\xi} .
\end{aligned}
$$

Neural networks approximate the nonlinear system as

$$
f_{1}+d_{a}=W_{1}^{* T} \Psi_{x}+\varepsilon_{x} .
$$

Based on the neural networks adaptive law (48) and the control design (47), we have

$$
\dot{\xi}_{2}=-\widetilde{W}_{1}^{T} \Psi_{x}+\varepsilon_{x}-\xi_{1}-a_{2} \xi_{2}^{p}-b_{2} \xi_{2}^{q} .
$$

If we choose the Lyapunov candidate function

$$
V_{1}=\frac{1}{2} \xi_{1}^{2}
$$

then we have

$$
\dot{V}_{1}=-a_{1} \xi_{1}^{p+1}-b_{1} \xi_{1}^{q+1}+\xi_{1} \xi_{2} .
$$

If we choose the Lyapunov candidate function

$$
V_{2}=\frac{1}{2} \xi_{2}^{2}
$$

then we have

$$
\dot{V}_{2}=-\xi_{2} \widetilde{W}_{1}^{T} \Psi_{x}+\varepsilon_{x} \xi_{2}-\xi_{1} \xi_{2}-a_{2} \xi_{2}^{p+1}-b_{2} \xi_{2}^{q+1} .
$$

If we choose the Lyapunov candidate function

$$
V_{N N X}=\frac{1}{2} \widetilde{W}_{1}^{T} \Gamma_{1}^{-1} \widetilde{W}_{1},
$$

then we have

$$
\dot{V}_{N N X}=\widetilde{W}_{1}^{T} \Gamma_{1}^{-1} \dot{\widetilde{W}}_{1}=\widetilde{W}_{1}^{T}\left(\Psi_{x} \xi_{2}-\sigma_{a x} \widehat{W}_{1}^{p}-\sigma_{b x} \widehat{W}_{1}^{q}\right) .
$$

If we choose the Lyapunov candidate function

$$
V=V_{1}+V_{2}+V_{N N X}
$$

$$
\begin{aligned}
\dot{V}= & -a_{1} \xi_{1}^{p+1}-b_{1} \xi_{1}^{q+1}+\varepsilon_{x} \xi_{2}-a_{2} \xi_{2}^{p+1}-b_{2} \xi_{2}^{q+1} \\
& -\sigma_{a x} \widetilde{W}_{1}^{T} \widehat{W}_{1}^{p}-\sigma_{b x} \widetilde{W}_{1}^{T} \widehat{W}_{1}^{q} .
\end{aligned}
$$

Based on Lemmas 3 and 4, the following inequations hold

$$
\begin{aligned}
\varepsilon_{x} \xi_{2} \leq & \frac{1}{q+1} \xi_{2}^{q+1}+\frac{q}{q+1} \varepsilon_{x}^{(q+1 / q)}-\sigma_{a x} \widetilde{W}_{1}^{T} \widehat{W}_{1}^{p} \\
\leq & -c_{p x}\left(\frac{1}{2} \widetilde{W}_{1}^{T} \Gamma_{1}^{-1} \widetilde{W}_{1}\right)^{(p+1 / 2)}+b_{p x} W_{1}^{*(p+1 / 2) T} W_{1}^{*(p+1 / 2)} \\
& -\sigma_{b x} \tilde{W}_{1}^{T} \widehat{W}_{1}^{q} \leq-c_{q x}\left(\frac{1}{2} \tilde{W}_{1}^{T} \Gamma_{1}^{-1} \tilde{W}_{1}\right)^{(q+1 / 2)} \\
& +b_{q x} W_{1}^{*(q+1 / 2) T} W_{1}^{*(q+1 / 2)} .
\end{aligned}
$$

Then, based on Lemma 3, we have

$$
\begin{aligned}
\dot{V} \leq & -a_{1} \xi_{1}^{p+1}-b_{1} \xi_{1}^{q+1}-a_{2} \xi_{2}^{p+1}-b_{2} \xi_{2}^{q+1}+\frac{1}{q+1} \xi_{2}^{q+1} \\
& +\frac{q}{q+1} \varepsilon_{x}^{(q+1 / q)}-c_{p x}\left(\frac{1}{2} \widetilde{W}_{1}^{T} \Gamma_{1}^{-1} \widetilde{W}_{1}\right)^{(p+1 / 2)} \\
& +b_{p x} W_{1}^{*(p+1 / 2) T} W_{1}^{*(p+1 / 2)} \\
& -c_{q x}\left(\frac{1}{2} \widetilde{W}_{1}^{T} \Gamma_{1}^{-1} \widetilde{W}_{1}\right)^{(q+1 / 2)}+b_{q x} W_{1}^{*(q+1 / 2) T} W_{1}^{*(q+1 / 2)} .
\end{aligned}
$$

Then, based on Lemma 2, we have

$$
\begin{aligned}
\dot{V} \leq & -2^{(p+1 / 2)} a_{1} V_{1}^{(p+1 / 2)}-2^{(q+1 / 2)} b_{1} V_{1}^{(q+1 / 2)}-2^{(p+1 / 2)} a_{2} V_{2}^{(p+1 / 2)} \\
& -2^{(q+1 / 2)}\left(b_{2}-\frac{1}{q+1}\right) V_{2}^{(p+1 / 2)}-c_{p x} V_{N N X}^{(p+1 / 2)}-c_{q x} V_{N N X}^{(q+1 / 2)} \\
& +\frac{q}{q+1} \varepsilon_{x}^{(q+1 / 2)}+b_{p x} W_{1}^{*(p+1 / 2) T} W_{1}^{*(p+1 / 2)} \\
& +b_{q x} W_{1}^{*(q+1 / 2) T} W_{1}^{*(q+1 / 2)} \\
\leq & -a_{x} V^{(p+1 / 2)}-b_{x} V^{(q+1 / 2)}+c_{x},
\end{aligned}
$$

where

$$
\begin{aligned}
a_{x}= & \min \left(2^{(p+1 / 2)} a_{1}, 2^{(p+1 / 2)} a_{2}, 2^{(p+1 / 2)} c_{p}\right), \\
b_{x}= & \min \left(2^{(q+1 / 2)} 3^{1-q} b_{1}, 2^{(q+1 / 2)} 3^{1-q}\left(b_{2}-\frac{1}{q+1}\right), 2^{(q+1 / 2)} 3^{1-q} c_{q}\right), \\
c_{x}= & \frac{q}{q+1} \varepsilon_{x}^{(q+1 / 2)}+b_{p x} W_{1}^{*(p+1 / 2) T} W_{1}^{*(p+1 / 2)} \\
& +b_{q x} W_{1}^{*(q+1 / 2) T} W_{1}^{*(q+1 / 2)} .
\end{aligned}
$$

then we have 
Therefore, based on Lemma 1, the closed-loop system is practically fixed-time stable, and convergence time $T_{x \max }=\left(2^{(3-p / 2)} /(1-p) a_{x}\right)+\left(2 /(q-1) b_{x}\right)$.

Step 2. There are six degrees of freedom, namely, $Q_{x}$, $Q_{y}$, and $Q_{z}$ and angles $\phi, \theta$, and $\psi$, where $Q_{x}, Q_{y}$, and $Q_{z}$ can be obtained by using $u_{s}$ which was designed by using (47) and $u_{1}=\sqrt{Q_{x}^{2}+Q_{y}^{2}+Q_{z}^{2}}$. The arbitrary desired yaw references $\psi_{d}$ can be designed in advance, and then based on the mathematic model (12), the desired roll and pitch references $\phi_{d}$ and $\theta_{d}$ can be generated as (49) and (50). Then, the rotational tracking errors control system can be controlled in fixed-time neural network adaptive control.

Therefore, neural network control for the rotational tracking errors control system (45) is designed as follows:

$$
\zeta_{1}=e_{p}
$$

Then, based on (45), we have

$$
\dot{\zeta}_{1}=-a_{3} \zeta_{1}^{p}-b_{3} \zeta_{1}^{q}+\zeta_{2}
$$

where

$$
\zeta_{2}=s_{2}-\alpha_{\zeta}
$$

and then we have

$$
\begin{aligned}
\dot{\zeta}_{2} & =B u_{r}+f_{2}+d_{p}-\ddot{p}_{d}-\dot{\alpha}_{\zeta} \\
& =B u_{r}+W_{2}^{* T} \Psi_{p}+\varepsilon_{p}-\ddot{p}_{d}-\dot{\alpha}_{\zeta} .
\end{aligned}
$$

Neural networks approximate the nonlinear system as

$$
f_{2}+d_{p}=W_{2}^{* T} \Psi_{p}+\varepsilon_{p} .
$$

Based on the neural networks adaptive law (53) and the control design (52), we have

$$
\dot{\zeta}_{2}=-\widetilde{W}_{2}^{T} \Psi_{p}+\varepsilon_{p}-\zeta_{1}-a_{4} \zeta_{2}^{p}-b_{4} \zeta_{2}^{q} .
$$

If we choose the Lyapunov candidate function

$$
V_{3}=\frac{1}{2} \zeta_{1}^{2}
$$

then we have

$$
\dot{V}_{3}=-a_{3} \zeta_{1}^{p+1}-b_{3} \zeta_{1}^{q+1}+\zeta_{1} \zeta_{2} .
$$

If we choose the Lyapunov candidate function

$$
V_{4}=\frac{1}{2} \zeta_{2}^{2}
$$

then we have

$$
\dot{V}_{4}=-\zeta_{2} \widetilde{W}_{2}^{T} \Psi_{p}+\varepsilon_{p} \zeta_{2}-\zeta_{1} \zeta_{2}-a_{4} \zeta_{2}^{p+1}-b_{4} \zeta_{2}^{q+1}
$$

If we choose the Lyapunov candidate function

$$
V_{N N}=\frac{1}{2} \widetilde{W}_{2}^{T} \Gamma_{2}^{-1} \widetilde{W}_{2}
$$

then we have

$$
\dot{V}_{N N P}=\widetilde{W}_{2}^{T} \Gamma_{2}^{-1} \dot{\tilde{W}}_{2}=\widetilde{W}_{2}^{T}\left(\Psi_{p} \zeta_{2}-\sigma_{a p} \widehat{W}_{2}^{p}-\sigma_{b p} \widehat{W}_{2}^{q}\right)
$$

If we choose the Lyapunov candidate function

$$
V=V_{3}+V_{4}+V_{N N P}
$$

then we have

$$
\begin{aligned}
\dot{V}= & -a_{3} \zeta_{1}^{p+1}-b_{3} \zeta_{1}^{q+1}+\varepsilon_{p} \zeta_{2}-a_{4} \zeta_{2}^{p+1}-b_{4} \zeta_{2}^{q+1} \\
& -\sigma_{a p} \widetilde{W}_{2}^{T} \widehat{W}_{2}^{p}-\sigma_{b p} \widetilde{W}_{2}^{T} \widehat{W}_{2}^{q} .
\end{aligned}
$$

Based on Lemmas 3 and 4, the following inequations hold:

$$
\begin{aligned}
\varepsilon_{p} \zeta_{2} \leq & \frac{1}{q+1} \zeta_{2}^{q+1}+\frac{q}{q+1} \varepsilon_{p}^{(q+1 / q)}-\sigma_{a p} \widetilde{W}_{2}^{T} \widehat{W}_{2}^{p} \\
\leq & -c_{p p}\left(\frac{1}{2} \widetilde{W}_{2}^{T} \Gamma_{2}^{-1} \widetilde{W}_{2}\right)^{(p+1 / 2)} \\
& +b_{p p} W_{2}^{*(p+1 / 2) T} W_{2}^{*(p+1 / 2)} \\
-\sigma_{b p} \widetilde{W}_{2}^{T} \widehat{W}_{2}^{q} \leq & -c_{q p}\left(\frac{1}{2} \widetilde{W}_{2}^{T} \Gamma_{2}^{-1} \widetilde{W}_{2}\right)^{(q+1 / q)} \\
& +b_{q p} W_{2}^{*(q+1 / q) T} W_{2}^{*(q+1 / q)} .
\end{aligned}
$$

Then, based on Lemma 3, we have

$$
\begin{aligned}
\dot{V} \leq & -a_{3} \zeta_{1}^{p+1}-b_{3} \zeta_{1}^{q+1}-a_{4} \zeta_{2}^{p+1}-b_{4} \zeta_{2}^{q+1}+\frac{1}{q+1} \zeta_{2}^{q+1} \\
& +\frac{q}{q+1} \varepsilon_{p}^{(q+1 / q)}-c_{p p}\left(\frac{1}{2} \widetilde{W}_{2}^{T} \Gamma_{2}^{-1} \tilde{W}_{2}\right)^{(p+1 / 2)} \\
& +b_{p p} W_{2}^{*(p+1 / 2) T} W_{2}^{*(p+1 / 2)} \\
& -c_{q p}\left(\frac{1}{2} \widetilde{W}_{2}^{T} \Gamma_{2}^{-1} \widetilde{W}_{2}\right)^{(q+1 / 2)}+b_{q p} W_{2}^{*(q+1 / 2) T} W_{2}^{*(q+1 / 2)} .
\end{aligned}
$$

Then, based on Lemma 2, we have

$$
\begin{aligned}
\dot{V} \leq & -2^{(p+1 / 2)} a_{3} V_{3}^{(p+1 / 2)}-2^{(q+1 / 2)} b_{3} V_{3}^{(q+1 / 2)} \\
& -2^{(p+1 / 2)} a_{4} V_{4}^{(p+1 / 2)}-2^{(q+1 / 2)}\left(b_{4}-\frac{1}{q+1}\right) V_{4}^{(q+1 / 2)} \\
& -c_{p p} V_{N N P}^{(p+1 / 2)}-c_{q p} V_{N N}^{(q+1 / 2)}+\frac{q}{q+1} \varepsilon_{p}^{(q+1 / 2)} \\
& +b_{p p} W_{2}^{*(p+1 / 2) T} W_{2}^{*(p+1 / 2)}+b_{q p} W_{2}^{*(q+1 / 2) T} W_{2}^{*(q+1 / 2)} \\
\leq & -a_{p} V^{(p+1 / 2)}-b_{p} V^{(q+1 / 2)}+c_{p},
\end{aligned}
$$

where 


$$
\begin{aligned}
a_{p}= & \min \left(2^{(p+1 / 2)} a_{3}, 2^{(p+1 / 2)} a_{4}, 2^{(p+1 / 2)} c_{p}\right), \\
b_{p}= & \min \left(2^{(q+1 / 2)} 3^{1-q} b_{3}, 2^{(q+1 / 2)} 3^{1-q}\left(b_{4}-\frac{1}{q+1}\right), 2^{(q+1 / 2)} 3^{1-q} c_{q}\right) \\
c_{p}= & \frac{q}{q+1} \varepsilon_{p}^{(q+1 / q)}+b_{p p} W_{2}^{*(p+1 / 2) T} W_{2}^{*(p+1 / 2)} \\
& +b_{q p} W_{2}^{*(q+1 / 2) T} W_{2}^{*(q+1 / 2)} .
\end{aligned}
$$

Therefore, based on Lemma 1, the closed-loop system is practically fixed-time stable, and convergence time $T_{p \max }=\left(2^{(3-p / 2)} /(1-p) a_{p}\right)+\left(2 /(q-1) b_{p}\right)$.

The proof for the theorem is completed.

\section{Simulation}

In this section, simulation is conducted to verify the effectiveness of the presented fixed-time adaptive neural network adaptive control for UAVs.

Example 1. Consider the UAV dynamic system (12) with the parameters as

$$
\begin{aligned}
& m=2, l=0.2, g=9.8, \\
& \xi_{x}=\xi_{x}=\xi_{x}=1.2, \\
& \xi_{\phi}=\xi_{\theta}=\xi_{x}=1.2, \\
& I_{x}=1.25, I_{y}=1.25, I_{z}=2.5 .
\end{aligned}
$$

The reference trajectory is selected as follows:

$$
\chi_{d}(t)=\left[5\left(1-\cos \left(\frac{\pi}{10} t\right)\right), 5 \sin \left(\frac{\pi}{10} t\right), 10\left(1-e^{-0.3 t}\right)\right]^{T} .
$$

In addition, the desired yaw angle is $\psi_{d}=0$.

Choose the parameters of controllers and NNs as

$$
\begin{aligned}
& a_{1}=a_{3}=b_{1}=b_{3}=1.1, \\
& a_{2}=a_{4}=b_{4}=b_{2}=1, \\
& \sigma_{a x}=\sigma_{b x}=\sigma_{a p}=\sigma_{b p}=1, \\
& \Gamma_{1}=\Gamma_{2}=I, p=\frac{1}{3}, q=3 .
\end{aligned}
$$

Based on such parameters and the fixed-time control result in Theorem 1, $T_{x \max }=T_{p \max }=5.3811$, which is independent with system initial conditions. Then, the virtual control, control input, and adaptive law of NNs are given as follows:

$$
\begin{aligned}
\alpha_{\xi} & =-1.1 \xi_{1}^{(1 / 3)}-1.1 \xi_{1}^{3}, \\
u_{s} & =2\left(-\widehat{W}_{1}^{T} \Psi_{x}-\ddot{x}_{d}+\dot{\alpha}_{\xi}-\xi_{1}-\xi_{2}^{(1 / 3)}-\xi_{2}^{3}\right), \\
\dot{\hat{W}}_{1} & =\Psi_{x} \xi_{2}-\widehat{W}_{1}^{(1 / 3)}-\widehat{W}_{1}^{3} .
\end{aligned}
$$

The initialization of the variable are selected as
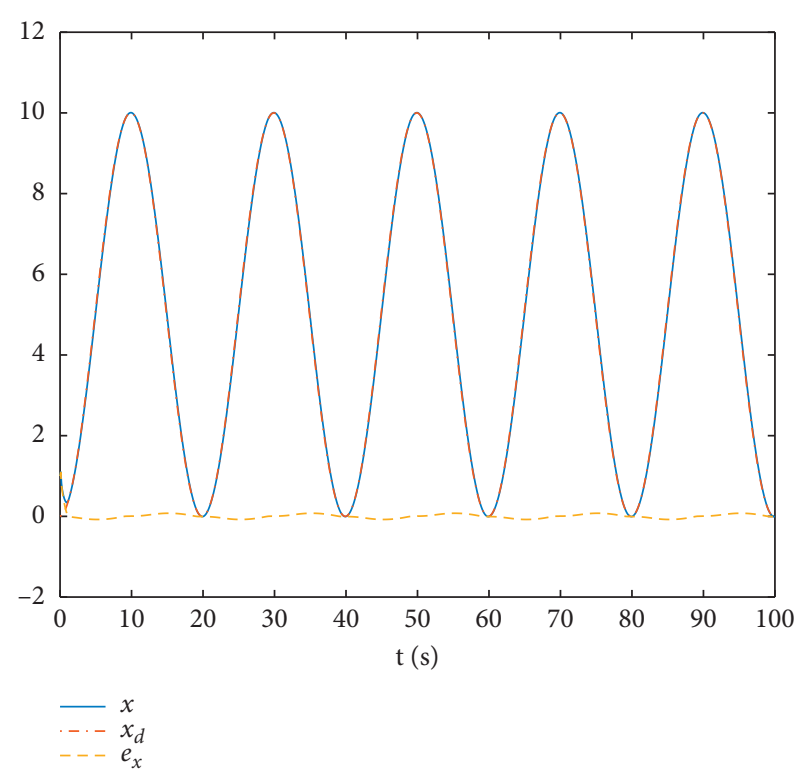

FIgURE 2: Trajectories of position $x$, ideal position $x_{d}$, and error $e_{x}$.

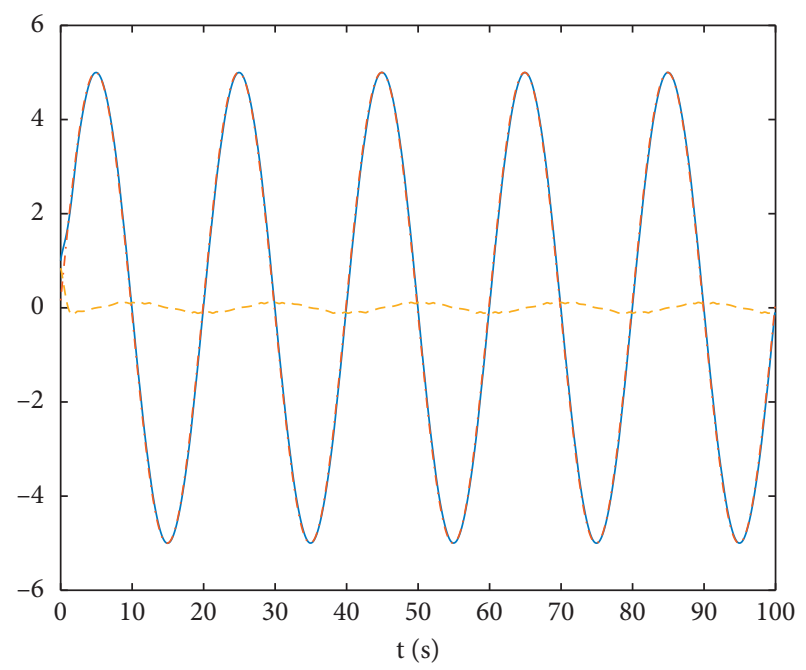

$y$
$-\cdot-y_{d}$
$---e_{y}$

FIgURE 3: Trajectories of position $y$, ideal position $y_{d}$, and error $e_{y}$.

$$
x(0)=y(0)=z(0)=\phi(0)=\theta(0)=\psi(0)=1 .
$$

The simulation results of the neural network controller are shown in Figures 2-9. The trajectories of positions $x, y$, and $z$ can be seen in Figures 2-4. The position tracking errors show that the proposed neural fixed-time control has good tracking performance, and based on parameters selected, the convergence time is $T_{x \max }=5.3811$. Figure 5 shows the 3-D moving trajectory by neural network control. The trajectories of attitudes $\phi, \theta$, and $\psi$ can be seen in Figures 6-8, which indicate better tracking performance of neural network control. The trajectories of NNs can be seen in Figure 9. The attitude tracking errors show that the 


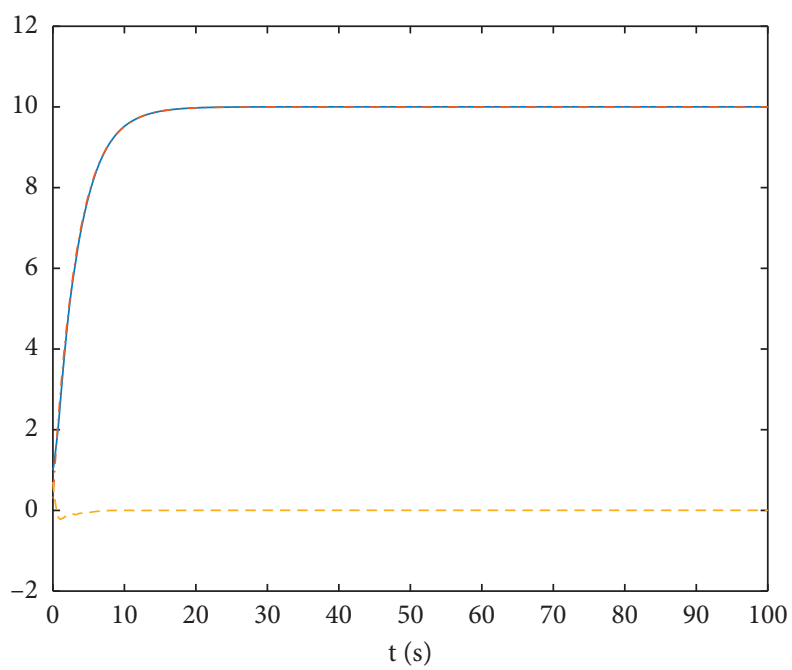

$\begin{array}{ll}z & z \\ -\cdots & z_{d} \\ --- & e_{z}\end{array}$

FIgURE 4: Trajectories of position $z$, ideal position $z_{d}$, and error $e_{z}$.

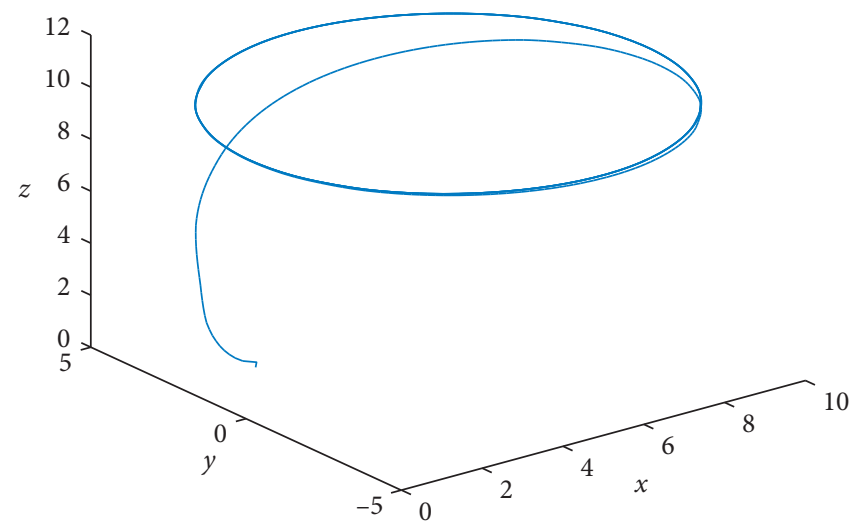

FIgURE 5: 3-D space state tracking result.

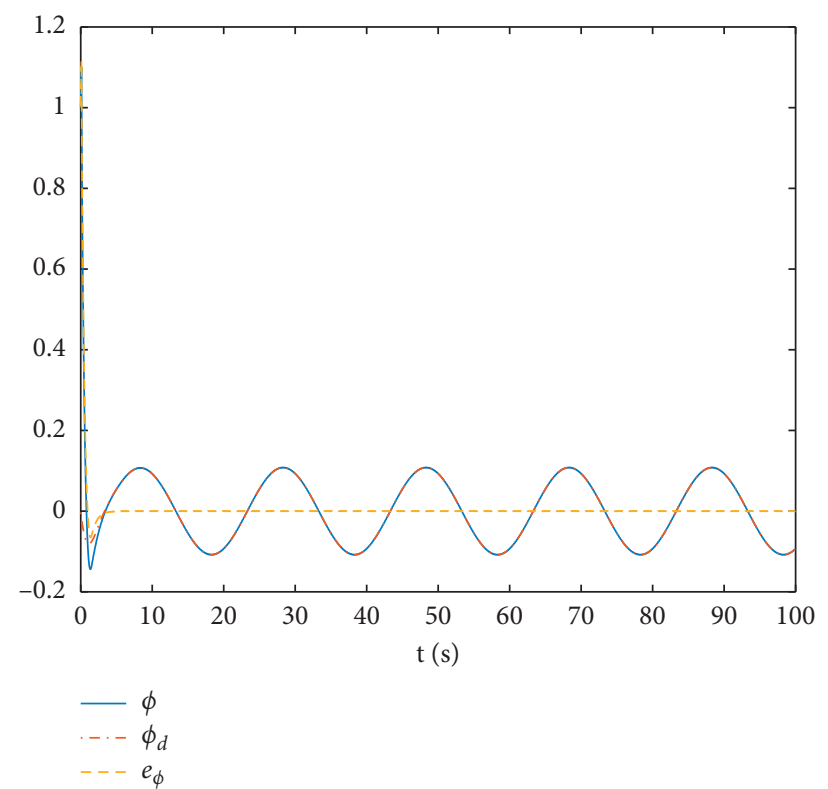

Figure 6: Trajectories of attitude $\phi$, ideal position $\phi_{d}$, and error $e_{\phi}$. 


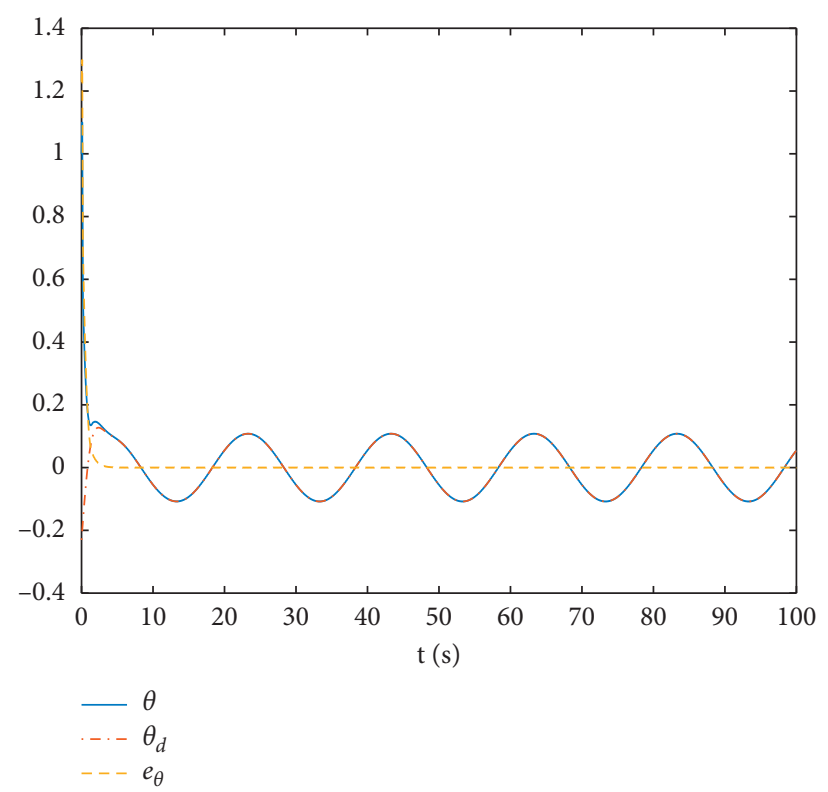

Figure 7: Trajectories of attitude $\theta$, ideal position $\theta_{d}$, and error $e_{\theta}$.

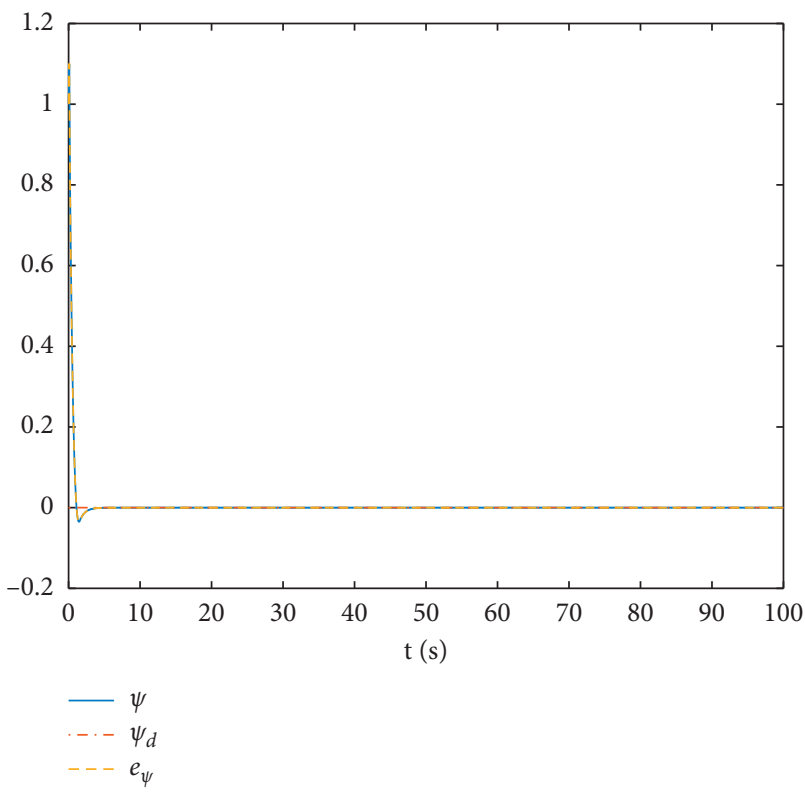

FIGURE 8: Trajectories of attitude $\psi$, ideal position $\psi_{d}$, and error $e_{\psi}$.

proposed neural fixed-time control has good tracking performance, and based on parameters selected, the convergence time is $T_{p \max }=5.3811$.

\section{Conclusion}

In this paper, based on the backstepping algorithm, fixedtime Lyapunov practical stable theory, and neural network adaptive control technology, a new fixed-time adaptive practical tracking control method is developed for quadrotor UAVs for the first time. The states of close-loop systems which contain error states and weights of the NNs are practically fixed-time stable, which are independent of states. Simulation result shows that the adaptive neural network

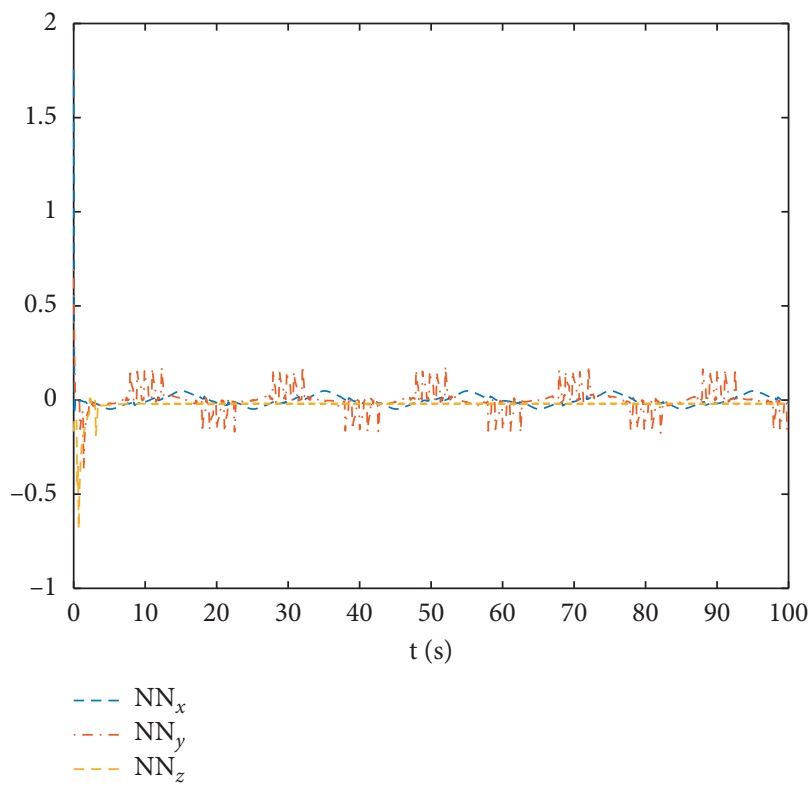

FIgURE 9: Trajectories of neural networks.

controller ensures stable position and attitude tracking of UAVs in finite time, which only depends on controller parameters. Bench simulation results showed the effectiveness of the adaptive fixed-time NN control method.

\section{Data Availability}

No data were used to support this study.

\section{Conflicts of Interest}

The funding did not lead to any conflicts of interest regarding the publication of this manuscript. There are no conflicts of interest in this study. 


\section{Acknowledgments}

This work was partially supported by the National Nature Science Foundation of China under grants 61273188 and 61473312 and Taishan Scholar Construction Engineering Special Funding, Shandong, China. This work was supported by Hebei Province Nature Fund (F2015208128) and Projects of Hebei Province Department of Education (QN20140157 and BJ2016020).

\section{References}

[1] J. Zhang, Q. Zhu, L. Yang, and X. Wu, "Homeomorphism mapping based neural networks for finite time constraint control of a class of nonaffine pure-feedback nonlinear systems," Complexity, vol. 2019, pp. 1-11, 2019.

[2] X. Luo, S. Ge, J. Wang, and X. Guan, "Time delay estimationbased adaptive sliding-mode control for nonholonomic mobile robots," International Journal of Applied Mathematics in Control Engineering, vol. 1, no. 1, pp. 1-8, 2018.

[3] S. Wang, H. Yu, X. Gao, and N. Wang, "Adaptive barrier control for nonlinear servomechanisms with friction compensation," Complexity, vol. 2018, pp. 1-10, 2018.

[4] Y. Du, J. Fang, and C. Miao, "Frequency-domain system identification of an unmanned helicopter based on an adaptive genetic algorithm," IEEE Transactions on Industrial Electronics, vol. 61, no. 2, pp. 870-881, 2014.

[5] Q. Zhu, W. Zhang, J. Zhang, and B. Sun, "U-neural networkenhanced control of nonlinear dynamic systems," Neurocomputing, vol. 352, pp. 12-21, 2019.

[6] S. Shi, M. Liu, and J. Ma, "Fractional-order sliding mode control for a class of nonlinear systems via nonlinear disturbance observer," International Journal of Applied Mathematics in Control Engineering, vol. 1, no. 1, pp. 103-110, 2018.

[7] A. Tayebi and S. McGilvray, "Attitude stabilization of a VTOL quadrotor aircraft," IEEE Transactions on Control Systems Technology, vol. 14, no. 3, pp. 562-571, 2006.

[8] J. Na, Y. Huang, X. Wu, S.-F. Su, and G. Li, “Adaptive finitetime fuzzy control of nonlinear active suspension systems with input delay," IEEE Transactions on Cybernetics, vol. 50, no. 6, pp. 2639-2650, 2020.

[9] S. Wang and J. Na, "Parameter estimation and adaptive control for servo mechanisms with friction compensation," IEEE Transactions on Industrial Informatics, vol. 16, no. 11, pp. 6816-6825, 2020.

[10] S. Wang, L. Tao, Q. Chen, J. Na, and X. Ren, "USDE-based sliding mode control for servo mechanisms with unknown system dynamics," IEEE/ASME Transactions on Mechatronics, vol. 25, no. 2, pp. 1056-1066, 2020.

[11] Q. Chen, H. Shi, and M. Sun, "Echo state network-based backstepping adaptive iterative learning control for strictfeedback systems: an error-tracking approach," IEEE Transactions on Cybernetics, vol. 50, no. 7, pp. 3009-3022, 2020.

[12] J. Na, B. Wang, G. Li, S. Zhan, and W. He, "Nonlinear constrained optimal control of wave energy converters with adaptive dynamic programming," IEEE Transactions on Industrial Electronics, vol. 66, no. 10, pp. 7904-7915, 2019.

[13] S. Wang, J. Na, and Y. Xing, "Adaptive optimal parameter estimation and control of servo mechanisms: theory and experiments," IEEE Transactions on Industrial Electronics, vol. 2020, p. 1, 2020.

[14] J. De Jesus Rubio, J. Humberto Perez Cruz, Z. Zamudio, and A. J. Salinas, "Comparison of two quadrotor dynamic models," IEEE Latin America Transactions, vol. 12, no. 4, pp. 531-537, 2014.

[15] L. L. Gomes, L. Leal, T. R. Oliveira, J. P. V. S. Cunha, and T. C. Revoredo, "Unmanned quadcopter control using a motion capture system," IEEE Latin America Transactions, vol. 14, no. 8, pp. 3606-3613, 2016.

[16] J. Li and Y. Li, "Dynamic analysis and PID control for a quadrotor," in Proceedings of the IEEE International Conference on Mechatronics and Automation, Beijing, China, August 2011.

[17] C. Rosales, S. Tosetti, C. Soria, and F. Rossomando, "Neural adaptive PID control of a quadrotor using EFK," IEEE Latin America Transactions, vol. 16, no. 11, pp. 2722-2730, 2018.

[18] B. Zhang, G. Yoon, and H. Lim, "Attitude control and altitude control of a four-rotor flying robot," in Proceedings of the 2018 14th International Conference on Natural Computation, Fuzzy Systems and Knowledge Discovery (ICNC-FSKD), Huangshan, China, July 2018.

[19] M. Ryll, H. H. Bülthoff, and P. R. Giordano, "A novel overactuated quadrotor unmanned aerial vehicle: modeling, control, and experimental validation," IEEE Transactions on Control Systems Technology, vol. 23, no. 2, pp. 540-556, 2015.

[20] M. J. Reinoso, L. I. Minchala, P. Ortiz, D. F. Astudillo, and D. Verdugo, "Trajectory tracking of a quadrotor using sliding mode control," IEEE Latin America Transactions, vol. 14, no. 5, pp. 2157-2166, 2016.

[21] L. Benziane, A. El Hadri, A. Seba, A. Benallegue, and Y. Chitour, "Attitude estimation and control using linearlike complementary filters: theory and experiment," IEEE Transactions on Control Systems Technology, vol. 24, no. 6, pp. 2133-2140, 2016.

[22] R. Zhang, Q. Quan, and K.-Y. Cai, "Attitude control of a quadrotor aircraft subject to a class of time-varying disturbances," IET Control Theory \& Applications, vol. 5, no. 9, pp. 1140-1146, 2011.

[23] S. Islam, P. X. Liu, and A. El Saddik, "Robust control of fourrotor unmanned aerial vehicle with disturbance uncertainty," IEEE Transactions on Industrial Electronics, vol. 62, no. 3, pp. 1563-1571, 2015.

[24] Y.-C. Choi and H.-S. Ahn, "Nonlinear control of quadrotor for point tracking: actual implementation and experimental tests," IEEE/ASME Transactions on Mechatronics, vol. 20, no. 3, pp. 1179-1192, 2015.

[25] D. Matouk, O. Gherouat, F. Abdessemed, and A. Hassam, "Quadrotor position and attitude control via backstepping approach," in Proceedings of the 2016 8th International Conference on Modelling, Identification and Control (ICMIC), Algiers, Algeria, November 2016.

[26] H. Alazki, A. G. Escobar, J. E. Valenzuela, and O. Garcia, "Embedded super twisting control for the attitude of a quadrotor," IEEE Latin America Transactions, vol. 14, no. 9, pp. 3974-3979, 2016.

[27] D. Cabecinhas, R. Naldi, C. Silvestre, R. Cunha, and L. Marconi, "Robust landing and sliding maneuver hybrid controller for a quadrotor vehicle," IEEE Transactions on Control Systems Technology, vol. 24, no. 2, pp. 400-412, 2016.

[28] P. Castillo, A. Dzul, and R. Lozano, "Real-time stabilization and tracking of a four rotor mini-rotorcraft," in Proceedings of the 2003 European Control Conference (ECC), Cambridge, UK, September 2003.

[29] F. Chen, Q. Wu, B. Jiang, and G. Tao, "A reconfiguration scheme for quadrotor helicopter via simple adaptive control and quantum logic," IEEE Transactions on Industrial Electronics, vol. 62, no. 7, pp. 4328-4335, 2015. 
[30] Y. Song, L. He, D. Zhang, J. Qian, and J. Fu, "Neuroadaptive fault-tolerant control of quadrotor UAVs: a more affordable solution," IEEE Transactions on Neural Networks and Learning Systems, vol. 30, no. 7, pp. 1975-1983, 2019.

[31] Y. Teng, B. Hu, Z. Liu, J. Huang, and Z. Guan, "Adaptive neural network control for quadrotor unmanned aerial vehicles," in Proceedings of the 2017 11th Asian Control Conference (ASCC), Gold Coast, Australia, December 2017.

[32] N. Mohajerin and S. L. Waslander, "Modelling a quadrotor vehicle using a modular deep recurrent neural network," in Proceedings of the 2015 IEEE International Conference on Systems, Man, and Cybernetics, Hong Kong, October 2015.

[33] Q. Lin, Z. Cai, Y. Wang, J. Yang, and L. Chen, "Adaptive flight control design for quadrotor UAV based on dynamic inversion and neural networks," in Proceedings of the 2013 Third International Conference on Instrumentation, Measurement, Computer, Communication and Control, Shenyang, China, September 2013.

[34] D. E. Chang and Y. Eun, "Global chartwise feedback linearization of the quadcopter with a thrust positivity preserving dynamic extension," IEEE Transactions on Automatic Control, vol. 62, no. 9, pp. 4747-4752, 2017.

[35] Y. Bai, X. Gong, Z. Hou, and Y. Tian, "Stability control of quad-rotor based on explicit model following with inverse model feedforward method," in Proceedings of the 2011 IEEE International Conference on Mechatronics and Automation, Beijing, China, August 2011.

[36] J. Zhang, Q. Zhu, and Li Yang, "Convergence time calculation for supertwisting algorithm and application for nonaffine nonlinear systems," Complexity, vol. 2019, pp. 1-15, Article ID 6235190, 2019.

[37] Q. Chen, Y. Wang, and Z. Hu, "Finite time synergetic control for quadrotor UAV with disturbance compensation," International Journal of Applied Mathematics in Control Engineering, vol. 1, no. 1, pp. 31-38, 2018.

[38] F. Chen, R. Jiang, K. Zhang, B. Jiang, and G. Tao, "Robust backstepping sliding-mode control and observer-based fault estimation for a quadrotor UAV," IEEE Transactions on Industrial Electronics, vol. 63, no. 8, pp. 5044-5056, 2016.

[39] J. Zhang, L. Yang, W. Fei, and X. Wu, "U-model based adaptive neural networks fixed-time backstepping control for uncertain nonlinear system," Mathematical Problems in Engineering, vol. 2020, Article ID 8302627, 2020.

[40] M. Chen, H. Wang, and X. Liu, "Adaptive fuzzy practical fixed-time tracking control of nonlinear systems," IEEE Transactions on Fuzzy Systems, vol. 2019, p. 1, 2019. 